\title{
A Comparative Study of Lateral Lumbar Interbody Fusion and Posterior Lumbar Interbody Fusion in Degenerative Lumbar Spondylolisthesis
}

\author{
Abhijit Y. Pawar ${ }^{1}$, Alexander P. Hughes ${ }^{2}$, Andrew A. Sama ${ }^{2}$, \\ Federico P. Girardi ${ }^{2}$, Darren R. Lebl' ${ }^{2}$, Frank P. Cammisa ${ }^{2}$ \\ ${ }^{I}$ Ruby Hall Clinic and Dr. D. Y. Patil Medical College, Pune, India \\ ${ }^{2}$ Department of Orthopedics, Hospital for Special Surgery, New York, NY, USA
}

Study Design: Level 4 retrospective review.

Purpose: To compare the radiographic and clinical outcomes between posterior lumbar interbody fusion (PLIF) and lateral lumbar interbody fusion (LLIF) with posterior segmental spinal instrumentation (SSI) for degenerative lumbar spondylolisthesis.

Overview of Literature: Both PLIF and LLIF have been performed for degenerative spondylolisthesis with good results, but no study has directly compared these two techniques so far.

Methods: The electronic medical and radiographic records of 78 matched patients were analyzed. In one group, 39 patients underwent PLIF with SSI at 41 levels (L3-4/L4-5), while in the other group, 39 patients underwent the LLIF procedure at 48 levels (L3-4/ L4-5). Radiological outcomes such as restoration of disc height and neuroforaminal height, segmental lumbar lordosis, total lumbar Iordosis, incidence of endplate fracture, and subsidence were measured. Perioperative parameters were also recorded in each group. Clinical outcome in both groups was assessed by the short form-12, Oswestry disability index and visual analogue scale scores. The average follow-up period was 16.1 months in the LLIF group and 21 months in the PLIF group.

Results: The restoration of disc height, foraminal height, and segmental lumbar lordosis was significantly better in the LLIF group $(p<0.001)$. The duration of the operation was similar in both groups, but the average blood loss was significantly lower in the LLIF group $(p<0.001)$. However, clinical outcome scores were similar in both groups.

Conclusions: Safe, effective interbody fusion can be achieved at multiple levels with neuromonitoring by the lateral approach. LLIF is a viable treatment option in patients with new onset symptoms due to degenerative spondylolisthesis who have had previous lumbar spine surgery, and it results in improved sagittal alignment and indirect foraminal decompression.

Keywords: Interbody fusion; Degenerative spondylolisthesis; Segmental spinal instrumentation

\section{Introduction}

Degenerative spondylolisthesis in adults is often characterized by loss of disc height across the affected segment and sagittal translation, resulting in central, lateral recess, and foraminal stenosis $[1,2]$. The indications for surgical management are persistent or recurrent neurogenic claudication, significant reduction in the quality of life despite

Received Nov 11, 2014; Revised Mar 8, 2015; Accepted Mar 22, 2015

Corresponding author: Abhijit Y. Pawar

Ruby Hall Clinic and Dr. D. Y. Patil Medical College, Pimpri, Pune 411001, India

Tel: +91-88-8870-0016, Fax: +91-20-2616-4529, E-mail: drabhijitpawar@gmail.com 
a trial of conservative management, and progressive neurologic deficits $[2,3]$. Decompression alone has a limited role in management and solid arthrodesis is necessary for long-term successful outcome $[4,5]$. The goal of surgical treatment for spondylolisthesis includes decompression of neural elements, restoration of the disc space height, and alignment and stabilization of the motion segment $[1,6]$.

Posterior lumbar interbody fusion (PLIF) was initially introduced by Cloward [7] in 1953. PLIF allows access to both the posterior and anterior sides from all posterior approach, and success rates of above $90 \%$ have been reported in degenerative disc disease and spondylolisthesis [3-5]. However, PLIF is relatively contraindicated in patients with severe osteoporosis due to the risk of subsidence $[4,8]$. It may also be technically challenging in the presence of conjoined nerve roots and in the upper lumbar spine (cranial to L2) because of the possibility of thecal sac retraction and iatrogenic injury to the conus $[9,10]$. Lateral lumbar interbody fusion (LLIF) is a relatively new technique and was first described by Ozgur et al. [11]. LLIF allows the surgeon to access the intervertebral space via a minimally invasive direct lateral approach through the psoas muscle. The advantage of LLIF over the traditional anterior approach is the avoidance of exposure of the abdominal viscera, large vessels, and sympathetic plexus. Injury to the nerve roots and dura, and perineural fibrosis, which can occur after PLIF or TLIF, are minimized with this technique $[11,12]$. LLIF has been utilized to treat a variety of pathologies including adult degenerative scoliosis, central and foraminal stenosis, spondylolisthesis, and adjacent segment degeneration $[12,13]$.

Both PLIF and LLIF have been performed for treating degenerative spondylolisthesis with good results, but no direct comparison between these two techniques has been made so far $[5,6,14]$. The purpose of this study was to compare the radiographic and clinical outcome scores of patients who underwent LLIF with posterior segmental spinal instrumentation (SSI) for degenerative lumbar spondylolisthesis (DS) with those of matched controls who underwent PLIF with SSI.

\section{Materials and Methods}

After receiving the Institutional Review Board approval, we retrospectively reviewed the electronic medical records between March 2006 and December 2009. Patients who underwent LLIF with SSI and had at least 1-year follow-up at Hospital for Special Surgery (New York) were identified, and the matched control group (PLIF with SSI) was identified with respect to age, body mass index (BMI), and diagnosis of DS (L3-4 and L4-5 levels or both). If patients who underwent LLIF with SSI did not have matched controls in the PLIF with SSI group, they were excluded from the study. We excluded patients who underwent interbody fusion at levels other than L3-4 and L4-5, patients who underwent stand-alone interbody fusion as well as patients who did not have spondylolisthesis on radiographs.

Finally-matched 39 pairs, total 78 patients (21 males and 57 females) were identified, and the electronic medical and radiographic records of these patients were analyzed. Eighteen patients in the LLIF group while six patients in the PLIF group had previous lumbar spine surgery. In group A, we had 39 patients who underwent LLIF at 48 levels, while in group B, we had 39 patients who underwent PLIF at 41 levels. Lumbar levels included in both groups were L3-4 (total 18 levels in group A vs. total 7 levels in group B) and L4-5 (total 30 levels in the group A vs. total 34 levels in group B). Nine patients in the LLIF group and four patients in the PLIF group underwent multiple level lumbar fusion.

\section{Surgical procedure}

LLIF procedure was performed with the standard minimally invasive lateral approach or mini open technique as described in the literature using the LLIF system (XLIF, NuVasive Inc., San Diego, CA, USA) or the COUGARsystem (COUGAR-Depuy Spine Inc., Raynham, MA, USA) [11]. Bone morphogenetic protein (BMP) was used in all cases of LLIF for interbody fusion. The PLIF procedure was performed using the standard technique as described in the literature with either one large cage or two small cages packed with bone graft depending on the surgeon's preference $[3,5]$. Posterior segmental instrumentation was performed in all cases. All cases had undergone either unilateral or bilateral posterior segmental instrumentation depending on the surgeon's preference. In each case, intraoperative electromyography probes and active-run electromyography were used to prevent injury to the neural structures.

For each group, radiological outcomes including restoration of disc height and neuroforaminal height, segmental lumbar lordosis, total lumbar lordosis, fusion rates, inci- 
Table 1. Comparison of LLIF (group A) versus PLIF (group B)

\begin{tabular}{|c|c|c|c|}
\hline Variable & LLIF (group A) & PLIF (group B) & $p$-value \\
\hline Total no. of level & 48 & 41 & \\
\hline Age at surgery (yr) & 59.03 & 57.77 & 0.6 \\
\hline Body mass index $\left(\mathrm{kg} / \mathrm{m}^{2}\right)$ & 29.60 & 29.37 & 0.9 \\
\hline Female sex & 31 & 26 & 0.8 \\
\hline Previous lumbar surgery & 18 & 6 & 0.03 \\
\hline Degenerative disc disease & 38 & 37 & 0.8 \\
\hline Degenerative scoliosis & 10 & 0 & 0.001 \\
\hline Anterior spondylolisthesis & 43 & 36 & 0.086 \\
\hline Lateral spondylolisthesis & 10 & 0 & 0.001 \\
\hline Multiple level & 9 & 4 & 0.2 \\
\hline L3-4 level fusion & 18 & 7 & $<0.001$ \\
\hline L4-5 level fusion & 30 & 34 & 0.17 \\
\hline Dural tear & 0 & 5 & 0.014 \\
\hline Duration of surgery (min) & 260.2 & 256.3 & 0.75 \\
\hline Blood loss during surgery (mL) & 438 & 750 & $<0.001$ \\
\hline \multicolumn{4}{|l|}{ Bone graft } \\
\hline Allograft & 1 & 12 & \\
\hline IC/autograft & 8 & 24 & \\
\hline Bone morphogenetic protein & 39 & 1 & \\
\hline Change in SF-12 (PCS) score ${ }^{\text {b) }}$ & 10.31 & 6.06 & 0.07 \\
\hline Change SF-12 (MCS) score & 7.41 & 4.13 & 0.2 \\
\hline Change Oswestry disability index score & -19.53 & -7.71 & 0.001 \\
\hline Change visual analogue scale score & -4.63 & -4.36 & 0.6 \\
\hline
\end{tabular}

LLIF, lateral lumbar interbody fusion; PLIF, posterior lumbar interbody fusion; IC, iliac crest; SF-12, the short form-12; PCS, physical component score; MCS, mental component score.

${ }^{a)}$ Mean value; ${ }^{\text {b) }}$ As compared to preoperative scores.

dence of endplate fracture, and subsidence were recorded and analyzed. Patients' perioperative parameters including incidence of dural tears, operation time, estimated blood loss, and length of hospital stay were compared, and the clinical outcomes were assessed by the short form-12 (SF12) and Oswestry disability index (ODI, back pain questionnaire) as well as visual analogue scale (VAS) clinical outcome scores preoperatively and postoperatively to assess the quality of life in patients and pain after the surgical procedure.

\section{Statistical analysis}

Data analysis was conducted using Microsoft Excel 2010 and SPSS ver. 14.0 (SPSS Inc., Chicago, IL, USA). SF-12 questionnaire data were analyzed using the Health Outcomes Scoring software 4.0. Values for continuous variables we reported as the mean and standard deviation. The Mann-Whitney $U$-test was used because of non-normally distributed data. For comparing patient groups, we used the chi-square test with a $p$-value $<0.05$ being considered statistically significant. Radiological data were analyzed with picture archiving and communication system.

\section{Results}

The average follow-up period was 16.1 months in group A and 21 months in group B. There was no difference in terms of age, gender, and BMI between the two groups (Table 1). Total 89 levels were fused in 78 patients. Forty- 
eight levels were fused in group A versus 41 levels in group B. The average number of levels fused was 1.2 in the LLIF group, while the average number of levels fused was 1.1 in the PLIF group. In the LLIF group, L3-4 level fusion was performed at 18 levels, while L4-5 level fusion was performed at 30 levels. In the LLIF group, nine patients underwent multiple level fusion (Fig. 1). In the PLIF group, L3-4 level fusion was performed at 7 levels, whereas L4-5 level fusion was performed at 34 levels. In the PLIF group, four patients underwent multiple level fusion. BMP was used in 39 patients of the LLIF group and in 12 patients of the PLIF group. Iliac crest autograft was used in eight patients of the LLIF group versus 24 patients of the PLIF group and allograft was used in one patient of LLIF group versus 12 patients of the PLIF group. Mean duration of surgery in the LLIF group was 260 minutes and the mean duration of surgery in the PLIF group was 256 minutes $(p=0.751)$. Five patients in the PLIF group had an intraoperative incidental durotomy compared to none of the patients in the LLIF group. Estimated blood loss in the LLIF group was $438 \mathrm{~mL}$ versus $751 \mathrm{~mL}$ in the PLIF group $(p<0.001)$. Radiological outcomes in the PLIF group and the LLIF group are presented and compared in Table 2.

Two patients had endplate fracture intraoperatively in the LLIF group compared with none of the patients in the PLIF group. However, based on the radiological data, subsidence was seen at 7 levels in the LLIF group at the final follow-up and at 11 levels in the PLIF group at the final follow-up. In the LLIF group, sensory deficit was observed in seven patients (18\%) and anterior groin pain and thigh pain were observed in eight patients $(21 \%)$, and psoas
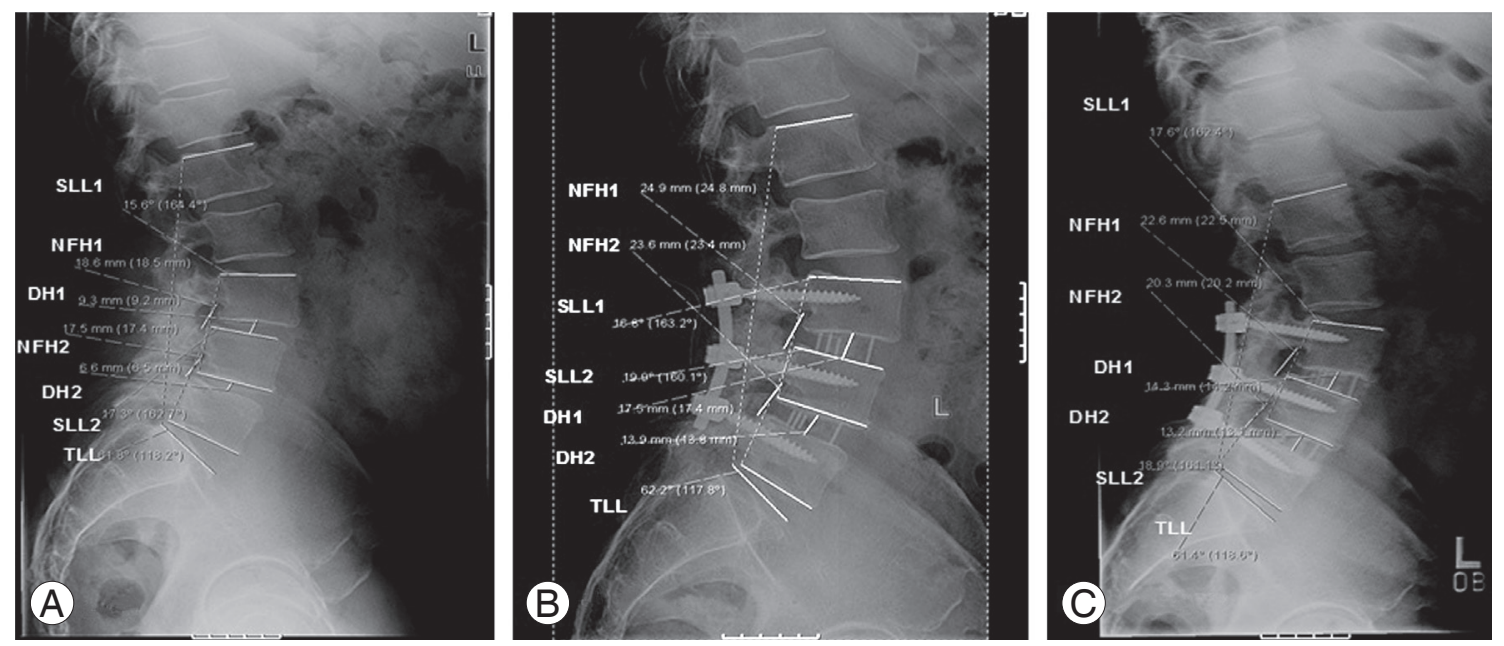

Fig. 1. A 57-year-old male with lumbar spinal stenosis and spondylolisthesis treated by lateral lumbar interbody fusion with posterior segmental spinal instrumentation. (A) Preoperative, (B) initial postoperative, (C) last postoperative. SLL, segmental lumbar lordosis; $\mathrm{NFH}$, neuroforaminal height; DH, disc height; TLL, total lumbar lordosis.

Table 2. Radiological outcomes in each group

\begin{tabular}{|c|c|c|c|c|c|c|}
\hline \multirow{2}{*}{$\begin{array}{l}\text { Serial } \\
\text { number }\end{array}$} & \multirow{2}{*}{ Variable } & \multicolumn{2}{|c|}{ Group A } & \multicolumn{2}{|c|}{ Group B } & \multirow{2}{*}{$p$-value } \\
\hline & & Preoperative & Final follow-up & Preoperative & Final follow-up & \\
\hline 1 & Disc height L3-4 (mm) & 7.4 & 13.2 & 9.6 & 10.5 & $<0.001$ \\
\hline 2 & Disc height L4-5 (mm) & 8.0 & 13.3 & 8.4 & 10.2 & $<0.001$ \\
\hline 3 & Neuroforaminal height L3-4 (mm) & 15.6 & 19.4 & 18.9 & 18.1 & $<0.001$ \\
\hline 4 & Neuroforaminal height L4-5 (mm) & 14.4 & 18.4 & 16.2 & 16.4 & $<0.001$ \\
\hline 5 & Segmental lordosis L3-4 $\left(^{\circ}\right)$ & 11.2 & 12.7 & 12.2 & 12.3 & 0.075 \\
\hline 6 & Segmental lordosis $L 4-5\left(^{\circ}\right)$ & 15.6 & 19.6 & 16.8 & 17.4 & $<0.001$ \\
\hline 7 & Lumbar lordosis L1-S1 $\left(^{\circ}\right)$ & 44.1 & 47.5 & 47.1 & 48.4 & 0.020 \\
\hline
\end{tabular}


mechanical flexion deficit was observed in five patients (13\%) at the first follow-up; however, none of the patients had sensory or motor deficits at the 1-year follow-up. None of the patients had lumbar plexus related deficits. In the PLIF group, seven patients (18\%) had radicular symptoms and nine patients (23\%) had dysesthesia at the first follow-up, while none of the patients had neurological symptoms at the 1-year follow-up. No permanent motor deficits were observed in patients of the PLIF group.

The patients filled in the SF-12 questionnaire, and there was an average improvement of 10.31 points in the physical component summary and of 7.41 points in the mental component summary in the LLIF group versus an average improvement of 6.06 and 4.13 points, respectively in the PLIF group. Both components showed higher quality of life in patients compared with the preoperative status. The difference between the two groups was not significant $(p>0.05)$. The ODI score also indicated better outcomes in both groups as compared to the preoperative status; however, a significantly better improvement was seen in the LLIF group (decrease of 19.53 points in the LLIF group vs. decrease of 7.71 points in the PLIF group, $p=0.001$ ). During the follow-up period, patients also filled in the VAS form to assess their pain. The average results at the 6-month follow-up showed better VAS scores in both groups (decrease of 4.63 points in the LLIF group vs. decrease of 4.36 points in the PLIF group).

\section{Discussion}

Lumbar fusion is indicated in degenerative spondylolisthesis to control pain due to segmental instability and mechanical insufficiency caused by degenerative changes $[1,2]$. The advantages of PLIF in treating these patients with DS are technical ease, allowance for direct decompression of the nerve root, restoration of disc height, increment of the size of the intervertebral neural foramen, and improvement in the load bearing ability of anterior ligaments and muscles [3,4]. PLIF is also more cost-effective than the anteroposterior 360-degree fusion [3]. LLIF is a minimally invasive surgical method which is used for operative stabilization of the lumbar spine. The mini-open technique offers a favorable safety profile due to rapid localization and preparation of the disc space $[12,15]$.

Biomechanical studies of different interbody devices have shown that interbody cages with larger area of contact between the cage and the end plate produce bony fusion with physiologic stress distribution $[4,8,11,16]$. Larger interbody cages for LLIF increase the surface area of bone graft; thus, creating a biomechanically superior environment for bone healing. Lateral fusion allows excellent disc space preparation, disc height and alignment restoration, better structural support to the apophyseal ring and decompression of the nerve roots by powerful indirect decompression $[11,14,17]$. This was evident in the radiological comparison in this study. In the current study, the average change in the disc space height at the L3-4 level and L4-5 level in the LLIF group (6 $\mathrm{mm}$ at L3 -4 and $5.5 \mathrm{~mm}$ at L4-5) was significantly greater as compared to that in the PLIF group (1.7 $\mathrm{mm}$ at L3-4 and $2 \mathrm{~mm}$ at L4-5, $p<0.001)$. Change in segmental lordosis at the L4-5 level (4 degrees) in the LLIF group was significant as compared to that in the PLIF group (0.7 degrees, $p<0.05)$. Change in total lumbar lordosis was 3.4 degrees in the LLIF group, while change in total lumbar lordosis was 1.3 degrees in the PLIF group; however, this difference was not significant. Mean change in the neuroforaminal height was $3.9 \mathrm{~mm}$ at the L3-4 level and $4 \mathrm{~mm}$ at the L4-5 level in the LLIF group, and it was significant as compared to that in the PLIF group $(p<0.001)$. Thus, LLIF improves the sagittal profile better as compared to PLIF as indicated by significantly better restoration of disc height, segmental lordosis and improved total lumbar lordosis [18]. This technique can be effective in patients with DS and flat back deformity. Injury to the nerve roots, dura, perineural fibrosis, and cage migration which could occur after PLIF or TLIF are minimized with this technique $[4,9]$. Sensory deficits and anterior thigh pain/groin pain were observed in $18 \%$ and $21 \%$ of the patients, respectively at the first follow-up visit in the current study; however, none of these patients had any residual deficits at the 1-year follow-up. Literature evidence suggests that the overall percentage of postoperative neurologic events ranges from $0.6 \%$ to $33.6 \%$ after LLIF $[12,19]$. Also, most of the sensory and motor deficits after LLIF are transient $[12,20,21]$. Authors of this study reported the incidence of neurologic deficit at the 12-month follow-up in a series of 235 patients with 444 levels treated by LLIF [20]. In that series, $1.6 \%$ of the patients had sensory deficit; $1.6 \%$ of the patients had psoas mechanical flexion deficit and $2.9 \%$ of the patients had lumbar plexus related deficits at the 1-year follow-up [20]. In another larger study in the literature, Rodgers et al. [12] reported that $0.7 \%$ of the patients had postoperative neurological deficits. 
In the current study, $18 \%$ of the patients in the PLIF group had some radicular symptoms and $23 \%$ of the patients had dysesthesia in the postoperative period, which resolved during the subsequent follow-up. In the series of 251 patients presented by Okuda et al. [3] in 2006, 6.7\% of the patients had neurologic injury resulting in dysesthesia and motor deficit after PLIF. In addition to these findings, five patients in the PLIF group had dural tears as compared none of the patients in the LLIF group, and subsidence was observed at 11 levels in the PLIF group at the final follow-up as compared to 7 levels in the LLIF group. LLIF seems to be better than PLIF because of decreased complication rate, increased versatility within the lumbar spine, and its minimally invasive technique. The patient outcome score including SF-12 (physical and mental component), VAS scores, and ODI scores improved in both groups at the final follow-up. The change in SF-12 and VAS scores was similar in both groups, while the improvement in the ODI score was significantly better in the LLIF group $(p=0.001)$. The reason for this variation in outcome scores is not clear.

The decision regarding the surgical approach is subjective and dependent on the patient's as well as the surgeon's preference, results of this study may be useful to make this decision after the attending physician discusses the drawbacks and advantages of both techniques. The current retrospective study is not without limitations. All surgeries were performed at a single institution by different surgeons. Although the patients were matched, surgeon bias could not be eliminated because treatment selection was not randomized. In general, the surgeons preferred the lateral approach if the patient had previous lumbar spine surgery and degenerative disc disease. The lower incidence of neurologic deficits in the current study after LLIF can also be attributed to the fact that the mini-open technique with self-lighted retractor system was used by four surgeons for gaining exposure in 36 cases. Single incision and percutaneous approach was used in three cases by one surgeon. Mini-open technique allows direct visualization of the lumbar nerves and minimizes the chances of injury; however, there is no definitive evidence to support this claim. Another limitation of the patient outcome scoring was that newly developing pain in the thigh and legs was recorded at the follow-up in both groups only if it was subjectively stated by the patient. This could have affected the outcome scores and the record of neurological complications at the final follow-up. A larger level 1 or
2 multicenter study with a longer follow-up can provide additional information on the optimal management of DS.

\section{Conclusions}

Minimally invasive interbody fusion with LLIF was associated with significantly lower estimated blood loss and reduced incidence of intraoperative complications as compared with PLIF. The radiological outcome was significantly better in the LLIF group than in the PLIF group; however, the clinical outcome was better in both groups at the final follow-up. Safer interbody fusion can be achieved at multiple levels with effective neuromonitoring by the LLIF technique. It is an effective option in adult patients with new onset axial low back pain and spondylolisthesis who have had previous lumbar spine surgery.

\section{Conflict of Interest}

No potential conflict of interest relevant to this article was reported.

\section{References}

1. Herkowitz HN. Degenerative lumbar spondylolisthesis: evolution of surgical management. Spine J 2009; 9:605-6.

2. Martin CR, Gruszczynski AT, Braunsfurth HA, Fallatah SM, O'Neil J, Wai EK. The surgical management of degenerative lumbar spondylolisthesis: a systematic review. Spine (Phila Pa 1976) 2007;32:1791-8.

3. Okuda S, Miyauchi A, Oda T, Haku T, Yamamoto T, Iwasaki M. Surgical complications of posterior lumbar interbody fusion with total facetectomy in 251 patients. J Neurosurg Spine 2006;4:304-9.

4. DiPaola CP, Molinari RW. Posterior lumbar interbody fusion. J Am Acad Orthop Surg 2008;16:130-9.

5. Yan DL, Pei FX, Li J, Soo CL. Comparative study of PILF and TLIF treatment in adult degenerative spondylolisthesis. Eur Spine J 2008;17:1311-6.

6. Molinari RW, Sloboda J, Johnstone FL. Are 2 cages needed with instrumented PLIF? A comparison of 1 versus 2 interbody cages in a military population. Am J Orthop (Belle Mead NJ) 2003;32:337-43.

7. Cloward RB. The treatment of ruptured lumbar intervertebral discs by vertebral body fusion. I: indi- 
cations, operative technique, after care. J Neurosurg 1953;10:154-68.

8. Rapoff AJ, Ghanayem AJ, Zdeblick TA. Biomechanical comparison of posterior lumbar interbody fusion cages. Spine (Phila Pa 1976) 1997;22:2375-9.

9. Chrastil J, Patel AA. Complications associated with posterior and transforaminal lumbar interbody fusion. J Am Acad Orthop Surg 2012;20:283-91.

10. Humphreys SC, Hodges SD, Patwardhan AG, Eck JC, Murphy RB, Covington LA. Comparison of posterior and transforaminal approaches to lumbar interbody fusion. Spine (Phila Pa 1976) 2001;26:567-71.

11. Ozgur BM, Aryan HE, Pimenta L, Taylor WR. Extreme lateral interbody fusion (XLIF): a novel surgical technique for anterior lumbar interbody fusion. Spine J 2006;6:435-43.

12. Rodgers WB, Gerber EJ, Patterson J. Intraoperative and early postoperative complications in extreme lateral interbody fusion: an analysis of 600 cases. Spine (Phila Pa 1976) 2011;36:26-32.

13. Isaacs RE, Hyde J, Goodrich JA, Rodgers WB, Phillips FM. A prospective, nonrandomized, multicenter evaluation of extreme lateral interbody fusion for the treatment of adult degenerative scoliosis: perioperative outcomes and complications. Spine (Phila Pa 1976) 2010;35(26 Suppl):S322-30.

14. Marchi L, Abdala N, Oliveira L, Amaral R, Coutinho E, Pimenta L. Stand-alone lateral interbody fusion for the treatment of low-grade degenerative spondylolisthesis. ScientificWorldJournal 2012;2012:456346.

15. Sharma AK, Kepler CK, Girardi FP, Cammisa FP,
Huang RC, Sama AA. Lateral lumbar interbody fusion. Clinical and radiographic outcomes at 1 year: a preliminary report. J Spinal Disord Tech 2011;24:24250.

16. Xu H, Tang H, Guan X, et al. Biomechanical comparison of posterior lumbar interbody fusion and transforaminal lumbar interbody fusion by finite element analysis. Neurosurgery 2013;72(1 Suppl Operative):21-6.

17. Oliveira L, Marchi L, Coutinho E, Pimenta L. A radiographic assessment of the ability of the extreme lateral interbody fusion procedure to indirectly decompress the neural elements. Spine (Phila $\mathrm{Pa} 1976$ ) 2010;35(26 Suppl):S331-7.

18. Le TV, Vivas AC, Dakwar E, Baaj AA, Uribe JS. The effect of the retroperitoneal transpsoas minimally invasive lateral interbody fusion on segmental and regional lumbar lordosis. ScientificWorldJournal 2012;2012:516706.

19. Youssef JA, McAfee PC, Patty CA, et al. Minimally invasive surgery. Lateral approach interbody fusion: results and review. Spine (Phila Pa 1976) 2010;35(26 Suppl):S302-11.

20. Pumberger M, Hughes AP, Huang RR, Sama AA, Cammisa FP, Girardi FP. Neurologic deficit following lateral lumbar interbody fusion. Eur Spine J 2012; 21:1192-9.

21. Kepler CK, Sharma AK, Huang RC, et al. Indirect foraminal decompression after lateral transpsoas interbody fusion. J Neurosurg Spine 2012;16:329-33. 